\title{
The relationship between vitronectin and hepatic insulin resistance in type 2 diabetes mellitus
}

\author{
Yan $\mathrm{Cao}^{1)}$ *, Xinyu $\mathrm{Li}^{1), 2)}$ *, Chong $\mathrm{Lu}^{3)}$ and Xiaorong Zhan ${ }^{1)}$ \\ 1) Department of Endocrinology, the First Affiliated Hospital of Harbin Medical University, Harbin, 150007, China \\ 2) Department of Pharmacology, the State-Province Key Laboratories of Biomedicine-Pharmaceutics of China, Key Laboratory of \\ Cardiovascular Research, Ministry of Education, Harbin Medical University, Harbin, Hei Long Jiang Province 150081, PR China \\ 3) Department of Neurology, Heilongjiang Provincial Hospital, Harbin, Hei Long Jiang Province 150036, PR China
}

\begin{abstract}
The World Health Organization (WHO) estimates that approximately 300 million people will suffer from diabetes mellitus by 2025. Type 2 diabetes mellitus (T2DM) is much more prevalent. T2DM comprises approximately $90 \%$ of diabetes mellitus cases, and it is caused by a combination of insulin resistance and inadequate compensatory insulin secretory response. In this study, we aimed to compare the plasma vitronectin (VN) levels between patients with T2DM and insulin resistance (IR) and healthy controls. Seventy patients with IR and 70 age- and body mass index (BMI)-matched healthy controls were included in the study. The insulin, Waist-to-Hip Ratio (WHR), C-peptide (CP) and VN levels of all participants were examined. The homeostasis model of assessment for insulin resistence index (HOMA-IR (CP)) formula was used to calculate insulin resistance. The levels of BMI, fasting plasma gluose (FPG), 2-hour postprandial glucose (2hPG), glycated hemoglobins (HbA1c), and HOMA-IR (CP) were significantly elevated in case group compared with controls. VN was found to be significantly decreased in case group. (VN Mean (Std): $8.55(2.92)$ versus $12.88(1.26) \mathrm{ng} / \mathrm{mL} p<0.001$ ). Multiple linear regression analysis was performed. This model explained $43.42 \%$ of the total variability of VN. Multiple linear regression analysis showed that HOMA-IR (CP) and age independently predicted VN levels. The VN may be a candidate target for the appraisal of hepatic insulin resistance in patients with T2DM.
\end{abstract}

Key words: Type 2 diabetes mellitus, Vitronectin, Hepatic insulin resistance, Age, C peptide

THE WORLD HEALTH ORGANIZATION (WHO) estimates that approximately 300 million people will suffer from diabetes mellitus by 2025 [1]. Type 2 diabetes mellitus (T2DM) is much more prevalent [2]. T2DM

Submitted Nov. 29, 2017; Accepted Apr. 5, 2018 as EJ17-0504

Released online in J-STAGE as advance publication May 18, 2018

Correspondence to: Xiaorong Zhan, Department of Endocrinology, the First Affiliated Hospital of Harbin Medical University, Youzheng road NO.23, Nangang district, Harbin, 150007 PR China.

E-mail: xiaorongzhanhrb@163.com

*Contributed equally

Abbreviations: ALT, Alanine aminotransferase; AST, Aspartate transaminase; BMI, Body mass index; BUN, Blood urea nitrogen; CP, C-peptide; DBP, Diastolic blood pressure; ELISA, EnzymeLinked ImmunoSorbent Assay; ECM, Extracellular matrix; FPG, fasting plasma gluose; GGT, Gamma-glutamyl transferase; HbAlc, glycated hemoglobins; HDL-C, High density lipoproteincholesterol; HOMA-IR, Homeostasis model of assessment for insulin resistence index; 2hPG, 2-hour postprandial glucose; LDLC, Low density lipoprotein-cholesterol; SBP, Systolic blood pressure; TC, Total cholesterol; TG, Triglyceride; UA, Uric acid; VN, Vitronectin comprises approximately $90 \%$ of diabetes mellitus cases, and it is caused by a combination of insulin resistance and inadequate compensatory insulin secretory response [3]. Insulin resistance plays a key role in the onset and progression of type 2 diabetes, and severe insulin resistance can lead to persistent beta cell function failure. The improvement in the insulin resistance of beta cells, "burden", contributes to prevent the failure of beta cells and restore their function, thereby delaying the onset and progression of T2DM. Therefore, the improvement of insulin resistance is required for T2DM prevention and treatment $[4,5]$.

The liver is the most important organ of insulin resistance, although the mechanism of hepatic insulin resistance remains unclear. Therefore, we used the RNA-seq method to detect differentially expressed genes (DEGs) between the type 2 diabetes cynomolgus model and the normal cynomolgus monkey liver. Then, we performed functional and pathway annotation of DEGs. There was a significant difference in ECM-receptor interaction 
between the normal group and the type 2 diabetes mellitus model. The results suggest that our ECM may be associated with hepatic insulin resistance [6].

The extracellular matrix (ECM) surrounding cells plays a key role in several biological and pathological processes, including inflammation, tissue remodeling, and invasion by cancer cells. Simultaneously, ECM is the microenvironment of the main cellular components, and it is also responsible for intracellular and intracellular signal transmission.

Vitronectin, a glycoprotein encoded by the VTN gene, is a cell adhesion factor found in the plasma and extracellular matrix (ECM) [7-10] Vitronectin is involved in various biological processes, including the regulation of coagulation pathways and the formation of the membrane attack complex (MAC), cell attachment and migration, and wound healing and tissue remodeling [11-13]. Vitronectin has been identified as a marker of profibrotic activity in several tissues, such as liver, heart and kidney [14-16]. It also has antimicrobial properties thanks to its heparin-binding domains $[17,18]$. Our previous studies have shown that vitronectin (VN) expression in the liver of macaques with type 2 diabetes mellitus is significantly lower than that of normal macaques [6]. Thus, we speculate that vitronectin may affect liver insulin signaling.

Although the VN effects on patient lungs [19] and tumor formation [20] has been investigated, no study on their relationship with T2DM, which is an IR disorder, has been published thus far. We aimed to compare the levels of $\mathrm{VN}$ between patients with T2DM who also have IR and healthy controls.

\section{Materials and Methods}

\section{Patients and healthy controls}

We recruited 70 healthy controls (33 males and 37 females) and 70 patients (43 males and 27 females) from the endocrinology department of the first affiliated hospital of Harbin Medical University, who met the inclusion criteria selected for this case study, between June and November 2016. The study was performed following approval by the ethics committee. Ethical review number: 201647.

\section{Inclusion criteria}

Inclusion criteria of the control group

(1) Age between 18-75 years old;

(2) BMI between $16 \mathrm{~kg} / \mathrm{m}^{2}$ and $45 \mathrm{~kg} / \mathrm{m}^{2}$;

(3) No other diseases or related drug history.

\section{Inclusion criteria of the test group}

(1) Presence of type 2 diabetes (according to the 2010 ADA diagnostic criteria for diabetes);

(2) Diabetes history less than 1 year;

(3) Presence of metabolic syndrome (according to 2007 Chinese Adult Blood Lipid Prevention Guide)

(4) Age between 18-75 years;

(5) BMI between $16 \mathrm{~kg} / \mathrm{m}^{2}$ and $45 \mathrm{~kg} / \mathrm{m}^{2}$

(6) Ability to express feelings in words

(7) Normal coagulation function

\section{Exclusion criteria}

(1) Presence of Type 1 diabetes mellitus;

(2) Recent occurrence of infectious disease or acute complications of diabetes, such as ketoacidosis and hypertonic coma;

(3) Presence of mental illness;

(4) Pregnant or lactating;

(5) Impaired function of critical organs such as heart, liver and kidney;

(6) Presence of malignant tumors;

(7) Presence of other hormonal diseases requiring long-term use of glucocorticoids;

(8) Presence of other medical diseases requiring longterm use of aspirin, Lipitor or any other drugs;

(9) Presence of hyperglycemia requiring exogenous insulin and any anti-diabetic medicines

\section{Research method and observation indices Basic data acquisition}

On the day of admission, a detailed history was taken, and a physical examination was performed, including age, course of the disease, smoking history, drinking history, history of hypertension, lifestyle and treatment, among others. The height and weight of the subjects were measured, and body mass index (BMI) was calculated. BMI $=$ weight $/$ height $^{2}\left(\mathrm{~kg} / \mathrm{m}^{2}\right)$. A measuring tape was used at the umbilical level to measure waist circumference $(\mathrm{W})$ and at the widest hip diameter to measure hip circumference $(\mathrm{H})$ to calculate the waist-to-hip ratio, according to the $\mathrm{W}$ and $\mathrm{H}$. The sitting, systolic (SBP) and diastolic (DBP) blood pressure values were measured at resting state.

\section{Biochemical indicator detection}

Hepatic and renal function, blood lipids and glycated hemoglobin (HbAlc) were assessed in the patients. Serum insulin and C-peptide were assessed by performing the C-peptide release test on the second day of admission. Venous plasma glucose and serum C-peptide 
levels were measured at fasting.

\section{Laboratory studies}

Hepatic and renal function and blood glucose and lipid levels were assessed at the clinical laboratory of the first affiliated hospital of Harbin medical university using an automated biochemistry analyzer and auxiliary reagents. C-peptide was detected by radioimmunoassay using a $\gamma$ ray counter, and the $\mathrm{HbAlc}$ was measured using the glycated hemoglobin test. These values were both detected at the endocrine laboratory of the first affiliated hospital of Harbin medical university.

\section{Insulin resistance index}

Insulin resistance index (homeostasis model assessment (HOMA) -insulin resistance): [HOMA-IR (CP) = $1.5+\mathrm{FPG}(\mathrm{mmol} / \mathrm{L}) \times \mathrm{CP}(\mathrm{ng} / \mathrm{mL}) / 2800][21,22]$.

\section{Detection of vitronectin ( $\mathrm{VN})$}

Blood samples were collected from each person from the antecubital vein. The samples were centrifuged to separate the plasma and stored for analysis at $80^{\circ} \mathrm{C}$. The vitronectin (VN) levels were measured using the enzyme-linked immunosorbent assay (ELISA) method (Blue Gene for life science, ShangHai).

\section{Data collection}

Related clinical data of 140 people were recorded, edited and outlined in an Excel spreadsheet. These data include the basic information, height, weight, BMI, waist-hip ratio, smoking history, drinking history, renal function, blood lipid levels, drug history, FPG, $2 \mathrm{hPg}$, HbA1c, serum C-peptide, HOMA-IR, and VN levels. Simultaneously, data error correction, missing data supplement and the generation of new variables was conducted, according to the actual circumstance of collected data for further data sorting.

\section{Statistical analysis}

Normal distribution of the data was tested using the Kolmogorov-Smirnov test. For continuous variables that showed normal distribution, independent samples $t$-test was used to compare the variable mean between the two groups. Wilcoxon signed-rank sum test was used to compare the variable distribution of the two groups, when the variable showed non-normality. All continuous variables were expressed as median, Q25-Q75, range, mean and standard deviation (SD). For categorical variables, Chisquared test or Fisher's exact test were performed to compare the rate between the two groups. The relationship between $\mathrm{VN}$ and other demographic and laboratory characteristics was evaluated by Spearman correlation analysis. Multiple linear regression analysis was performed to adjust the covariates and identify independent relationships between VN levels and Sex, CHD, Smoke, Drink, Age, FPG, 2hPG, Cr, HbAlc, WHR, BMI and HOMA-IR. All independent variables in the multiple linear regression analysis were tested for multicollinearity. If the variance inflation factor (VIF) exceeded 2.5, the variable was considered collinear. All reported confidence interval (CI) values are calculated at the $95 \%$ level. A two-sided $p$ value $<0.05$ was considered significant. All analyses were performed using SAS version 9.4 (SAS Institute, Cary, NC)

\section{Results}

\section{Clinical and laboratory characteristics of the study population}

The comparative demographic and laboratory parameters of the study subjects in the two groups are outlined in Table 1 (for continuous variables) and Table 2 (for categorical variables). The levels of BMI, FPG, $2 \mathrm{hPG}$, $\mathrm{HbA} 1 \mathrm{c}$, and HOMA-IR were significantly elevated in the case group compared with the control group. VN was significantly decreased in the case group.

\section{Correlation of $V N$ with clinical parameters}

We then investigated the relationship between circulating $\mathrm{VN}$ levels and various other parameters in the control and case groups. Correlation coefficients are outlined in Table 3. VN levels were significantly correlated with FPG and the HOMA-IR in the control group. VN levels were significantly correlated with age in the case group.

\section{Multivariate binary logistic regression analysis}

Multiple linear regression analysis was performed (Table 4). This model explained $43.42 \%$ of the total variability of VN. Multiple linear regression analysis showed that HOMA-IR and age independently predicted the VN levels.

\section{Discussion}

Many studies supporting the crucial role of $\mathrm{VN}$ in the T2DM with IR were found in the literature [23]. This is the first time reported the describing the circulating VN levels in patients with T2DM and IR to our knowledge. In the present study, we found that circulating VN levels were significantly lower in patients with T2DM and IR than in healthy controls. Logistic regression analysis showed that low VN levels were significantly associated with high T2DM and IR disease ratios. In addition, 
Table 1 Comparison of the demographic and laboratory characteristicsof the subjects (continuous variables)

\begin{tabular}{|c|c|c|c|c|}
\hline Variables & & $\begin{array}{l}\text { Control } \\
N=70\end{array}$ & $\begin{array}{c}\text { Case } \\
N=70\end{array}$ & $p^{*}$ \\
\hline \multirow{4}{*}{ Age (Year) } & Median & 54.50 & 48.50 & \multirow{4}{*}{$0.2141^{\dagger}$} \\
\hline & Q25, Q75 & $38.00,63.00$ & $41.00,58.00$ & \\
\hline & Range & $22.00,77.00$ & $18.00,81.00$ & \\
\hline & Mean (Std) & $50.89(14.53)$ & $48.26(13.65)$ & \\
\hline \multirow{4}{*}{ BMI $\left(\mathrm{Kg} / \mathrm{m}^{2}\right)$} & Median & 24.95 & 27.35 & \multirow{4}{*}{0.0002} \\
\hline & Q25, Q75 & $22.60,26.90$ & $25.10,29.06$ & \\
\hline & Range & $16.60,36.62$ & $20.51,40.00$ & \\
\hline & Mean (Std) & $25.16(3.50)$ & $27.62(4.11)$ & \\
\hline \multirow{4}{*}{$\mathrm{Cr}(\mathrm{umol} / \mathrm{L})$} & Median & 61.95 & 63.10 & \multirow{4}{*}{$0.8840^{\dagger}$} \\
\hline & Q25, Q75 & $52.00,73.60$ & $52.30,68.20$ & \\
\hline & Range & $36.60,170.20$ & $36.00,92.70$ & \\
\hline & Mean (Std) & $65.42(21.38)$ & $62.38(12.74)$ & \\
\hline \multirow{4}{*}{ FPG (mmol/L) } & Median & 5.20 & 7.53 & \multirow{4}{*}{$<.0001$} \\
\hline & Q25, Q75 & $4.90,5.60$ & $6.30,9.10$ & \\
\hline & Range & $4.50,6.30$ & $4.54,14.90$ & \\
\hline & Mean (Std) & $5.25(0.43)$ & $7.82(1.92)$ & \\
\hline \multirow{4}{*}{$2 \mathrm{hPG}(\mathrm{mmol} / \mathrm{L})$} & Median & 7.55 & 10.90 & \multirow{4}{*}{$<.0001^{\S}$} \\
\hline & Q25, Q75 & $6.80,8.20$ & $9.80,12.80$ & \\
\hline & Range & $5.80,9.80$ & $5.20,22.20$ & \\
\hline & Mean (Std) & $7.46(0.94)$ & $11.42(2.91)$ & \\
\hline \multirow{4}{*}{ HbAlc (\%) } & Median & 5.10 & 7.55 & \multirow{4}{*}{$<.0001^{t}$} \\
\hline & Q25, Q75 & $4.90,5.40$ & $6.50,9.20$ & \\
\hline & Range & $3.90,6.10$ & $5.20,17.60$ & \\
\hline & Mean (Std) & $5.14(0.43)$ & $8.08(2.13)$ & \\
\hline \multirow{4}{*}{ HOMA-IR (CP) } & Median & 1.50 & 1.51 & \multirow{4}{*}{$<.0001^{\sharp}$} \\
\hline & Q25, Q75 & $1.50,1.50$ & $1.51,1.51$ & \\
\hline & Range & $1.50,1.51$ & $1.50,1.52$ & \\
\hline & Mean (Std) & $1.50(0.00)$ & $1.51(0.00)$ & \\
\hline \multirow{4}{*}{$\mathrm{VN}$} & Median & 12.91 & 7.99 & \multirow{4}{*}{$<.0001$} \\
\hline & Q25, Q75 & $12.29,13.40$ & $6.28,10.47$ & \\
\hline & Range & $7.60,16.08$ & $3.69,15.61$ & \\
\hline & Mean (Std) & $12.88(1.26)$ & $8.55(2.92)$ & \\
\hline \multirow{4}{*}{ WHR } & Median & 0.82 & 0.82 & \multirow{4}{*}{$0.2931^{\ddagger}$} \\
\hline & Q25, Q75 & $0.78,0.85$ & $0.76,0.84$ & \\
\hline & Range & $0.70,0.96$ & $0.65,0.92$ & \\
\hline & Mean (SD) & $0.82(0.05)$ & $0.80(0.06)$ & \\
\hline
\end{tabular}

\footnotetext{
$\dagger$ Independent samples $t$-test was performed;

$\S$ Satterthwaite $t$-test was performed;

\$ Wilcoxon rank sum test was performed;

* A $p$ value of $<0.05$ was considered significant.
} 
Table 2 Comparison of the demographic and clinical characteristicsof the subjects (binary variables)

\begin{tabular}{lccc}
\hline Variables & $\begin{array}{c}\text { Control } N=70 \\
N(\%)\end{array}$ & $\begin{array}{c}\text { Case } N=70 \\
N(\%)\end{array}$ & $p$ \\
\hline Sex (male) & $33(47.14)$ & $42(60.00)$ & $0.1272^{\ddagger}$ \\
CHD (Yes) & $11(15.71)$ & $15(21.43)$ & $0.3847^{\ddagger}$ \\
Smoke (Yes) & $20(28.57)$ & $22(31.43)$ & $0.7122^{\dagger}$ \\
Drink (Yes) & $17(24.29)$ & $16(22.86)$ & $0.8422^{\dagger}$ \\
\hline
\end{tabular}

$\dagger$ Fisher's exact test was performed;

$\$$ Chi-square test was performed;

Table 3 Spearman correlation coefficient between Vn levels and clinical parameters

\begin{tabular}{lcc}
\hline Variables & $\begin{array}{c}\text { Control Coefficient } \\
(p \text { value })\end{array}$ & $\begin{array}{c}\text { Case Coefficient } \\
(p \text { value })\end{array}$ \\
\hline Age $($ Year $)$ & $-0.20(0.0946)$ & $0.28(0.0171)$ \\
\hline BMI $\left(\mathrm{Kg} / \mathrm{m}^{2}\right)$ & $0.18(0.1338)$ & $-0.09(0.4603)$ \\
Cr $(\mathrm{umol} / \mathrm{L})$ & $-0.10(0.3901)$ & $-0.01(0.9535)$ \\
\hline FPG $(\mathrm{mmol} / \mathrm{L})$ & $-0.32(0.0069)$ & $-0.15(0.2160)$ \\
\hline hPG $(\mathrm{mmol} / \mathrm{L})$ & $0.08(0.5200)$ & $-0.08(0.5035)$ \\
\hline HbA1c $(\%)$ & $-0.19(0.1149)$ & $-0.11(0.3722)$ \\
\hline HOMA-IR $(\mathrm{CP})$ & $-0.30(0.0130)$ & $-0.20(0.0954)$ \\
\hline WHR & $-0.02(0.8514)$ & $-0.15(0.2141)$ \\
\hline
\end{tabular}

multivariate regression analysis showed that Sex, CHD, Smoke, Drink, Age, FPG, 2hPG, Cr, HbAlc, WHR, BMI and HOMA-IR (CP) were other independent factors affecting the serum $\mathrm{VN}$ levels.

Insulin resistance can be observed in patients with low VN levels who have a considerably higher risk for type 2 diabetes than healthy subjects. The autocrine and paracrine regulatory functions of $\mathrm{VN}$ on glucose homeostasis were defined in recent studies.

We used the RNA-seq technique to sequence and compare the transcriptomes of high-fat-induced T2DM cynomolgus monkeys and of cynomolgus monkeys fed a normal diet, and we found that 1,451 genes were differentially expressed (914 genes were up-regulated, and 537 genes were down-regulated). We performed the functional annotation of these differentially expressed genes and found that 48 genes were involved in extracellular matrix (ECM)-related processes $(p<0.05)$. We also analyzed the 1,451 differentially expressed genes by KEGG pathway annotation and found that the interaction between vitronectin and its receptor in the extracellular matrix is also one of the key pathways that shows a significant difference $(p<0.05)$. Thus, we speculate that extracellular matrix components of hepatocytes may be involved in the development of type 2 diabetes mellitus [6].

This experiment does not conflict with the findings of Alessi et al. They conducted 52-year follow-up experiments on 5,212 subjects aged 30-65 years between 1994 and 1996 into the D.E.S.I.R., a nine-year French prospective study aimed at clarifying the development of the insulin resistance syndrome. The results showed that VN has a strong correlation with PAI-1, and jointly affected the development of metabolic syndrome, insulin resistance, etc. To determine whether $\mathrm{VN}$ is an independent risk factor for T2DM insulin resistance, we established strict criteria for inclusion and exclusion. In order to determine whether $\mathrm{VN}$ is an independent risk factor for T2DM insulin resistance, we established strict entry criteria and exclusion criteria. A control group with a history of $<1$ year of new-onset diabetes and impaired glucose tolerance with defined insulin resistance was selected, and coagulation function, a variety of hypoglycemic agents and exogenous insulin and other effects were excluded. Different enrollment and exclusion criterias may lead to different results.

In international studies, Vuori $\mathrm{K}$ et al. [24] incubated culture human insulin receptor (HIRCB) cells with VN and found that the cell membrane insulin receptor substrate-1 (IRS-1) was activated, and that the intracellular integrin $\alpha \mathrm{v} \beta 3$ DNA levels were significantly increased. These results suggested that $\mathrm{VN}$ and its receptor integrin $\alpha v \beta 3$ have a direct role in the insulin signaling pathway IRS-1, an intracellular protein that binds to other signaling molecules to regulate insulin signaling [25]. However, the exact mechanism remains unclear.

In this study, we directly measured the human plasma concentration of vitronectin. The levels of vitronectin were compared between the healthy controls and type 2 diabetes mellitus group using statistical methods. The results showed that the levels of BMI, FPG, 2hPG, HbA1c and HOMA-IR (CP) were significantly elevated in the case group compared with the control group. VN was significantly decreased in the case group. VN levels were significantly correlated with age and the HOMA-IR (CP) in the case group. Multiple linear regression analysis showed that HOMA-IR (CP) and age independently predicted the VN levels. However, further studies are needed to assess the exact role of $\mathrm{VN}$ in the pathogenesis of T2DM and IR and to identify the initiating factor. 
Table 4 Evaluation of the effects of multiple variables on $\mathrm{VN}$ level using the multiple linear regression analysis $(R 2=0.4342)$

\begin{tabular}{lrrrrrr}
\hline Parameter & Estimate & StdErr & $t$-Value & $p$-value & LowerCL & UpperCL \\
\hline Age (Year) & 0.04 & 0.02 & 2.75 & $0.0069^{*}$ & 0.01 & 0.08 \\
\hline Sex (male) & -0.30 & 0.53 & -0.56 & 0.5775 & -1.36 & 0.76 \\
BMI (Kg/m²) & 0.00 & 0.07 & 0.03 & 0.9756 & -0.13 & 0.13 \\
\hline Cr (umol/L) & -0.00 & 0.01 & -0.01 & 0.9948 & -0.03 & 0.03 \\
FPG (mmol/L) & -0.45 & 0.27 & -1.66 & 0.0984 & -0.98 & 0.08 \\
\hline 2hPG (mmol/L) & -0.05 & 0.14 & -0.33 & 0.7426 & -0.33 & 0.23 \\
HbA1c (\%) & -0.09 & 0.21 & -0.43 & 0.6663 & -0.51 & 0.32 \\
HOMA-IR (CP) & -321.44 & 120.21 & -2.67 & $0.0085 *$ & -559.32 & -83.56 \\
WHR & 0.16 & 4.35 & 0.04 & 0.9710 & -8.46 & 8.78 \\
\hline Drink (Yes) & -0.19 & 0.55 & -0.35 & 0.7279 & -1.28 & 0.90 \\
Smoke (Yes) & -1.09 & 0.56 & -1.96 & 0.0518 & -2.20 & 0.01 \\
\hline CHD (Yes) & -0.78 & 0.55 & -1.43 & 0.1553 & -1.86 & 0.30 \\
\hline
\end{tabular}

Multiple linear regression analysis was used. $\beta$, unstandardized regression coefficient; $\mathrm{CI}$, confidence interval; BMI, body mass index; HOMA-IR (CP), homeostasis model assessment of insulin resistance;

*A $p$ value of $<0.05$ was considered significant.

To determine whether $\mathrm{VN}$ is an independent risk factor for T2DM insulin resistance, this experiment excluded the influence of complex factors such as coagulation function, duration of diabetes and medication history on the experimental results, and established strict entry criteria and exclusion criteria. Due to the limitation of the experiment duration, the sample size is small, the measurement index is relatively simple, and there is no long-term follow-up comparison, and a further research is required.

\section{Conclusion}

The level of vitronectin in patients with type 2 dia- betes was significantly decreased than in the normal control group, suggesting that vitronectin may be a new indicator of the degree of insulin resistance in patients with type 2 diabetes mellitus. HOMA-IR (CP) and age independently predicted the VN levels. The age and HOMA-IR (CP) were negatively correlated with vitronectin, suggesting that vitronectin and the formation of insulin resistance in type 2 diabetes is relevant.

\section{Disclosure of Conflicts of Interest}

The authors declare no conflicts of interest regarding the publication of this paper.

\section{References}

1. Siddiqui S (2014) Depression in type 2 diabetes mellitus —a brief review. Diabetes Metab Syndr 8: 62-65.

2. Modesti PA, Castellani S, Calabrese M, Malandrino D, Zhao D (2017) Comparison of type 2 diabetes prevalence in Chinese migrants vs. Caucasians and new perspectives for screening of cerebrovascular disease in Chinese: a proof of concept study. Diabetes Res Clin Pract 130: 196203.

3. Tangvarasittichai S (2015) Oxidative stress, insulin resist- ance, dyslipidemia and type 2 diabetes mellitus. World $J$ Diabetes 6: 456-480.

4. Duan Y, Liu J, Xu Y, Yang N, Yang W, et al. (2018) Factors that influence pancreatic beta cell function and insulin resistance in newly diagnosed type 2 diabetes patients: a sub-analysis of the MARCH trial. Diabetes Ther 9: 743752.

5. Reutrakul S, Van Cauter E (2018) Sleep influences on obesity, insulin resistance, and risk of type 2 diabetes. 
Metabolism [Epub ahead of print].

6. Li X, Lin Z, Zhan X, Gao J, Sun L, et al. (2018) RNA-seq analysis of the transcriptome of the liver of cynomolgus monkeys with type 2 diabetes. Gene 651: 118-125.

7. Barnes DW, Silnutzer J, See C, Shaffer M (1983) Characterization of human serum spreading factor with monoclonal antibody. Proc Natl Acad Sci U S A 80: 1362-1366.

8. Barnes DW, Silnutzer J (1983) Isolation of human serum spreading factor. J Biol Chem 258: 12548-12552.

9. Hanson B, Westin C, Rosa M, Grier A, Osipovitch M, et al. (2014) Estimation of protein function using templatebased alignment of enzyme active sites. BMC Bioinformatics 15: 87.

10. Suzuki S, Oldberg A, Hayman EG, Pierschbacher MD, Ruoslahti E (1985) Complete amino acid sequence of human vitronectin deduced from cDNA. Similarity of cell attachment sites in vitronectin and fibronectin. EMBO J 4 : 2519-2524.

11. Preissner KT (1989) The role of vitronectin as multifunctional regulator in the hemostatic and immune systems. Blut 59: 419-431.

12. Preissner KT, Jenne D (1991) Structure of vitronectin and its biological role in haemostasis. Thromb Haemost 66: 123-132.

13. Preissner KT, Seiffert D (1998) Role of vitronectin and its receptors in haemostasis and vascular remodeling. Thromb Res 89: 1-21.

14. Inuzuka S, Ueno T, Tanikawa K (1994) Fibrogenesis in acute liver injuries. Pathol Res Pract 190: 903-909.

15. Koukoulis GK, Shen J, Virtanen I, Gould VE (2001) Vitronectin in the cirrhotic liver: an immunomarker of mature fibrosis. Hum Pathol 32: 1356-1362.

16. Niculescu F, Rus HG, Vlaicu R (1987) Immunohistochemical localization of C5b-9, S-protein, C3d and apolipoprotein B in human arterial tissues with atheroscle- rosis. Atherosclerosis 65: 1-11.

17. Andersson E, Rydengard V, Sonesson A, Morgelin M, Bjorck L, et al. (2004) Antimicrobial activities of heparinbinding peptides. Eur J Biochem 271: 1219-1226.

18. Malmsten M, Davoudi M, Schmidtchen A (2006) Bacterial killing by heparin-binding peptides from PRELP and thrombospondin. Matrix Biol 25: 294-300.

19. Salazar-Pelaez LM, Abraham T, Herrera AM, Correa MA, Ortega JE, et al. (2015) Vitronectin expression in the airways of subjects with asthma and chronic obstructive pulmonary disease. PLoS One 10: e0119717.

20. Pirazzoli V, Ferraris GM, Sidenius N (2013) Direct evidence of the importance of vitronectin and its interaction with the urokinase receptor in tumor growth. Blood 121: 2316-2323.

21. Törn C, Landin-Olsson M, Lernmark A, Palmer JP, Arnqvist HJ, et al. (2000) Prognostic factors for the course of beta cell function in autoimmune diabetes. J Clin Endocrinol Metab 85: 4619-4623.

22. Li X, Zhou ZG, Qi HY, Chen XY, Huang G (2004) Replacement of insulin by fasting C-peptide in modified homeostasis model assessment to evaluate insulin resistance and islet beta cell function. Zhong Nan Da Xue Xue Bao Yi Xue Ban 29: 419-423 (In Chinese).

23. Alessi MC, Nicaud V, Scroyen I, Lange C, Saut N, et al. (2011) Association of vitronectin and plasminogen activator inhibitor-1 levels with the risk of metabolic syndrome and type 2 diabetes mellitus. Results from the D.E.S.I.R. prospective cohort. Thromb Haemost 106: 416-422.

24. Vuori K, Ruoslahti E (1994) Association of insulin receptor substrate-1 with integrins. Science 266: 1576-1578.

25. Copps KD, White MF (2012) Regulation of insulin sensitivity by serine/threonine phosphorylation of insulin receptor substrate proteins IRS1 and IRS2. Diabetologia 55: 2565-2582. 